OPEN ACCESS

Edited by:

Osama Mohamed Ahmed,

Beni-Suef University, Egypt

Reviewed by:

Rimke Vos,

Leiden University Medical

Center, Netherlands

James Lenhard,

Christiana Care Health System,

United States

*Correspondence:

Hasniza Zaman Huri

hasnizazh@um.edu.my

Specialty section:

This article was submitted to

Clinical Diabetes,

a section of the journal

Frontiers in Endocrinology

Received: 26 April 2020

Accepted: 08 June 2020

Published: 10 July 2020

Citation:

Lin SN, Phang KK, Toh SH, Chee KH and Zaman Huri $H$ (2020) Heart

Failure With Type 2 Diabetes Mellitus:

Association Between

Antihyperglycemic Agents, Glycemic

Control, and Ejection Fraction.

Front. Endocrinol. 11:448.

doi: $10.3389 /$ fendo.2020.00448

\section{Heart Failure With Type 2 Diabetes Mellitus: Association Between Antihyperglycemic Agents, Glycemic Control, and Ejection Fraction}

\author{
Shu Ning Lin ${ }^{1}$, Kok Kit Phang ${ }^{2}$, Seng Hsiung Toh ${ }^{3}$, Kok Han Chee ${ }^{1}$ and \\ Hasniza Zaman Huri ${ }^{4 *}$
}

${ }^{1}$ Department of Medicine, Faculty of Medicine, University of Malaya, Kuala Lumpur, Malaysia, ${ }^{2}$ Department of Medicine, Hospital Tengku Ampuan Rahimah, Ministry of Health Malaysia, Klang, Malaysia, ${ }^{3}$ Department of Cardiology, Hospital Queen Elizabeth II, Ministry of Health, Kota Kinabalu, Malaysia, ${ }^{4}$ Department of Clinical Pharmacy and Pharmacy Practice, Faculty of Pharmacy, University of Malaya, Kuala Lumpur, Malaysia

Background: Heart failure (HF) is associated with type 2 diabetes mellitus (T2DM). Antihyperglycemic drugs have interaction with heart failure among diabetic patients. To date, the data on real world use of diabetic medication in Malaysian heart failure patients with T2DM has not been elucidated.

Objective: This study aims to identify the prescribing pattern of antihyperglycemic regimens in HF patients with T2DM, and to investigate the association between glycemic control and other factors such as demographic and clinical characteristics with left ventricular ejection fraction (LVEF) in these patients.

Methods: This retrospective observational study involved patients diagnosed to have HF and T2DM who were seen in the outpatient clinic in a government tertiary hospital in Malaysia. Patients receiving at least one oral antidiabetic agent and/or insulin for at least 3 months prior were included. The differences and association between study outcomes were examined and analyzed using Pearson's Chi-square test, One-Way ANOVA, Binary Logistic Regression and multiple Multinomial Logistic Regression models.

Results: From July to December 2019, 194 patients were included in this study. The majority (52.1\%) of the patients had HF with preserved ejection fraction (HFpEF), 20.6\% had HF with mid-range EF (HFmrEF), and $27.3 \%$ had HF with reduced EF (HFrEF). Overall, metformin (59.8\%) was the commonest antihyperglycemic agent prescribed, followed by insulins (54.0\%), and sulphonylureas (44.9\%). The most prescribed agents for HFpEF, HFmrEF, and HFrEF patients were metformin (65.3\%), insulins (62.5\%), and sulphonylureas (60.4\%), respectively. The prescribing trend of sulphonylureas was found to be significantly associated with patients' LVEF status $(p=0.033)$. The odds for sulphonylurea prescription among the HFrEF patients were 2.42 times higher compared to the HFpEF patients [95\% confidence interval [Cl], 1.23-4.79]. There was no association found between glycemic control with patients' LVEF. 
Conclusion: Our findings reported metformin as the most commonly prescribed antihyperglycemic agent, sodium glucose linked transporter-2 (SGLT-2) inhibitor being under-prescribed, and detected poorly controlled diabetes in majority of patients with T2DM and HF. Understanding the prescribing pattern of antihyperglycemic agents supports the implementation of evidence-based treatment in HF patients with T2DM to improve patients' outcomes.

Keywords: antihyperglycemic agent, glycemic control, ejection fraction, heart failure, diabetes

\section{INTRODUCTION}

Heart Failure (HF) is a progressive and chronic clinical syndrome characterized by typical symptoms of breathlessness, orthopnea, or ankle swelling, caused by a structural or functional cardiac abnormality, resulting in a decreased cardiac output and raised intracardiac pressures at rest or during stress (1). Heart failure is a global pandemic affecting at least 26 million people worldwide and is increasing in prevalence (2). The prevalence of heart failure ranges between 3 and 20 per 1,000 population (3).

Type 2 diabetes mellitus (T2DM) is a prevalent noncommunicable disease characterized by hyperglycemia resulting from the combination of defects in insulin secretion, resistance to insulin action, and excessive glucagon secretion. The prevalence of T2DM has increased by $30 \%$ globally in the past decade (2). In Malaysia, the National Health and Morbidity Survey (NHMS) 2015 has reported an overall diabetes prevalence of $17.5 \%$ for adults above the age of 18 years, detecting an increment in the prevalence compared with $15.2 \%$ in the year $2011(4,5)$.

T2DM is a well-established risk factor for cardiovascular disease, with heart failure being tightly-linked to T2DM (6). Data from The Framingham Heart Study highlighted that the risks of heart failure are 2- and 5-fold, respectively, in men and women with diabetes (7). Data from the OPTIMIZE-HF and EVEREST studies also reported that $\sim 40 \%$ of patients hospitalized with heart failure had a diagnosis of diabetes $(8,9)$.

Classification of HF patients according to left ventricular ejection fraction (LVEF) has been shown to have prognostic significance. Heart failure has been categorized into three subtypes: HF with preserved Ejection Fraction (HFpEF) patients with LVEF $\geq 50 \%$, HF with mid-range Ejection Fraction (HFmrEF) patients with LVEF 40-49\%, and HF with reduced Ejection Fraction (HFrEF) patients with LVEF $<40 \%$ (1). LVEF is an established efficacy measure to predict major adverse cardiac events (MACE) (10). A 3\% improvement in LVEF was shown to correlate with a $20 \%$ improvement in mortality (11). Patients in different classes of LV myocardial dysfunction were also reported to respond to therapies differently. While the 1-year mortality rate after risk-adjustments appeared comparable among patients in different LVEF categories, heart failure patients with increasing New York Heart Association (NYHA) functional class face increased morbidity risk. NYHA functional classification is used to grade and describe the severity of symptoms and exercise tolerance of heart failure patients (1). Higher NYHA classes are associated with increased 1-year mortality rate. The 1-year mortality is estimated to be $5-10,10-15,15-20$, and $20-50 \%$, respectively, for patients with heart failure NYHA I, II, III, and IV (3).

Poor glycemic control and insulin resistance are associated with deterioration of heart failure and LV dysfunction (2). However, available data suggest no difference in the risk of worsening heart failure between subjecting patients to intensive glycemic control and standard treatment arms (2). The relationship between glycated hemoglobin (Hbalc) and LVEF remains unclear. The U-shaped relationship between $\mathrm{HbAlc}$ and mortality among HF patients does not translate into precise glycemic targets or ranges recommended for this group of patient (2).

When choosing appropriate antihyperglycemic therapies in patients with essential comorbidities such as heart failure, a patient-centered approach should be emphasized. Metformin have been shown to be safe and effective. On the contrary, insulin and sulphonylurea have been associated with worsening of heart failure (1). Thiazolidinediones have also demonstrated a consistent and robust relationship with increased risk of heart failure (12). Pooled analysis of currently available Cardiovascular Outcome Trials (CVOTs) of glucose-lowering medications reported that dipeptidyl peptidase-4 inhibitor (DPP4i) and GLP1-RA resulted in a neutral effect on heart failure hospitalization in patients with T2DM. In contrast, sodium glucose linked transporter-2 (SGLT-2) inhibitor resulted in a statistically significant reduction in heart failure hospitalization (13). While suggesting that metformin and sulphonylurea being generally safe, the Malaysian Clinical Practice Guideline (CPG) on the Management of Heart Failure (HF) also recommends SGLT-2 inhibitor in the treatment of diabetes in patients with $\operatorname{HF}(3)$.

The current published CVOTs of antihyperglycemic agents involve a large number of subjects from Asians, Caucasians, and Blacks patients, across different countries. Translating clinical trial data to real world practice, especially in resource-limited countries or hospitals, remains a challenge. The data on real world use of diabetic medicine in Asian HF patients with T2DM are not well-documented. This underscores the importance of seeking a better understanding of ethnicity-tailored treatment strategies for heart failure patients with T2DM.

Thus, a localized clinical observational study is required to identify the prescribing pattern of different classes of antihyperglycemic therapies, as well as to examine the effect of patients' glycemic control status on patients' cardiac function, in a real-world population of patients with both the diseases. This 
study aims to assess the prescribing pattern of antihyperglycemic regimens in heart failure patients with type 2 diabetes mellitus, and to investigate the association between glycemic control and other factors such as demographic and clinical characteristics, with LVEF of these patients.

\section{METHODOLOGY}

\section{Study Design and Setting}

This retrospective observational study was conducted at Hospital Tengku Ampuan Rahimah (HTAR) Klang, a government tertiary hospital with 831 beds. HTAR caters to a multi-ethnic society that makes up the population of Malaysia. The study was conducted in compliance with the ethical principles outlined in the Declaration of Helsinki and Malaysian Good Clinical Practical Guideline. Ethical approval has been obtained from the Medical Research and Ethics Committee (MREC), Ministry of Health Malaysia [Reference number NMRR-19-1358-48105 (IIIR)]. The MREC of $\mathrm{MOH}$ waived the need for written informed consent from the participants.

\section{Inclusion and Exclusion Criteria}

All eligible patients who fulfill the following inclusion and exclusion criteria were included in this study. The inclusion criteria were patients aged $\geq 18$ years old, diagnosed to have heart failure based on clinical diagnosis with echocardiographic evidence of ejection fraction $\leq 70 \%$, diagnosed with type 2 diabetes mellitus with laboratory measured $\mathrm{HbAlc}$ results, and prescribed with at least one oral antidiabetic agent and/or insulin and receiving antidiabetic therapy for at least 3 months prior to enrolment. The exclusion criteria were patients without an echocardiography report and $\mathrm{HbA} 1 \mathrm{c}$ results, T2DM patients who were not receiving any antidiabetic agent and managed with diet controls and lifestyle modification, and pregnant women.

\section{Study Procedure}

The potential patients who were coming for follow-up in the Medical Outpatients Clinic, HTAR from July to December 2019 were identified from MOPD Registry. During the patients' visit, an initial screening through patients' medical records for diagnosis of heart failure was conducted. Patients' echocardiography reports were retrieved from Echocardiogram Registry, with echocardiogram tests performed as early as 1 week prior to patients' scheduled clinic appointments. Patients diagnosed with both HF with left ventricular ejection fraction LVEF of $\leq 70 \%$ and T2DM were selected.

Data that were collected from patients' medical records included demographic information such as age, sex, ethnicity, weight, height, clinical characteristics including medical history, comorbidities, duration of diabetes, duration of heart failure, LVEF, NYHA classification, relevant laboratory results such as Hbalc, and lastly, medication history, current prescribed diabetes medication regimen, and other concurrent medications.

Finally, patients who fulfilled the inclusion and exclusion criteria were identified and were included in the study, with the summarized findings presented in Figure 1. Patients were then classified according to their LVEF into HF with preserved ejection fraction (HFpEF), HF with mid-range LVEF (HFmrEF), and $\mathrm{HF}$ with reduced Ejection Fraction (HFrEF).

Relevant data extracted from medical records were manually entered into the data collection forms. This was done with the permission from the treating physicians, with patients' identities remaining anonymous.

\section{Study Outcomes}

The primary outcome was to identify the prescribing pattern of antihyperglycemic regimens across the three patient groups, which are HFpEF patients, HFmrEF patients, and HFrEF patients. The secondary outcomes were to determine if there was an association between glycemic control and other patient characteristics with LVEF in HF patients with T2DM.

\section{Sampling Size}

The sample size required for this study was estimated using OpenEpi, Version 3.01 (14). Assuming 95\% confidence interval or $5 \%$ level of significance and $5 \%$ margin of error, the minimum sample size calculated was 139 .

\section{Statistical Techniques}

Data obtained were analyzed using Statistical Package for Social Science (SPSS) software Version 23 (IBM Corp., Armonk, N.Y., USA). Normality test was done using the Kolmogorov-Smirnov test. Continuous data were presented in mean and standard deviation (SD) if normally distributed, and in median with 25$75 \%$ percentile values if non-normally distributed. Categorical data were reported in frequency and percentage. Pearson's Chisquare test was used to study the association between categorical variables. The One-Way ANOVA procedure was used to test for difference in characteristics in the three groups of patients. Odds ratio [95\% confidence intervals [CI]] was computed using Binary Logistic Regression with dummy variable. Multiple Multinomial Logistic Regression models were conducted to test the association between antihyperglycemic agent and patients' characteristics with LVEF, adjusting for age, sex, and HbA1c. Significance level was set at $p<0.05$.

\section{RESULTS}

\section{Selection of Study Subjects}

As illustrated in Figure 1, there were a total of 1,108 heart failure patients who came for follow-up from July-Dec 2019. Out of the $976 \mathrm{HF}$ patients who had echocardiographic evidence of ejection fraction $\leq 70,20.8 \%$ had a concomitant diagnosis of T2DM. Nine patients were excluded from the study for not fulfilling the inclusion criteria. Therefore, a final total number of 194 patients were included in the study.

\section{Demographic and Clinical Characteristics}

Majority (52.1\%) of the patients had HFpEF, 20.6\% had HFmrEF, and $27.3 \%$ had HFrEF. The study population consisted of nearly three-quarters of male. As shown in Table 1, patients' age was normally distributed with a mean \pm standard deviation (SD) of $59.6 \pm 12.0$ years, with a minimum age of 25 and maximum age of 88 years old. LVEF was found to increase with advancing age. 
Approval from the MREC, MOH obtained

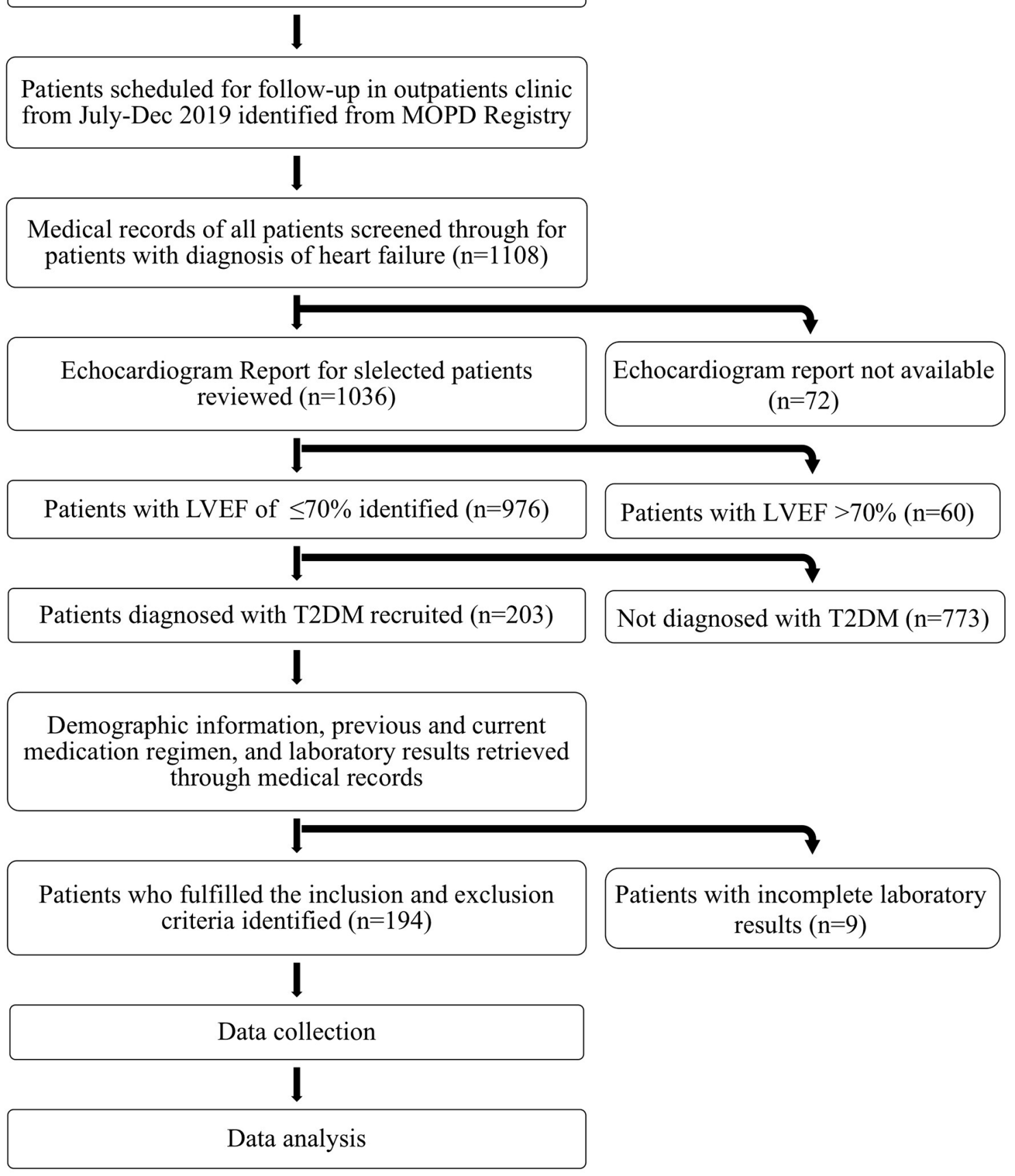

FIGURE 1 | Overview of study design and study subjects' selection.

Patients from HFrEF group had the youngest mean age (57.8 \pm 10.9 years). The largest ethnic population in this study was Malay, followed by Indian, Chinese, and others. When we compared between the three LVEF subgroups, there was significant sex disparity in the population of HF patients with T2DM. More than $80 \%$ of male patients were in the HFmrEF and HFrEF categories; while there were more than twice as many females in the HFpEF groups in comparison with HFmrEF or HFrEF groups. Sex was the only characteristic found to be significantly associated with LVEF status. Male HF patients were significantly more likely to have LVEF of $\leq 50 \%$ ( $p=0.002)$. There were no significant association between other demographic characteristics such as age and ethnicity with LVEF status across the three patient groups (Table 1). 
TABLE 1 | Demographic and clinical characteristics of patients.

\begin{tabular}{|c|c|c|c|c|c|}
\hline $\begin{array}{l}\text { Demographic and } \\
\text { clinical } \\
\text { characteristics }\end{array}$ & $\begin{array}{c}\text { Total } \\
n=194\end{array}$ & $\begin{array}{l}\text { HFpEF } \\
n=101 \\
(52.1 \%)\end{array}$ & $\begin{array}{l}\text { HFmrEF } \\
n=40 \\
(20.6 \%)\end{array}$ & $\begin{array}{l}\text { HFrEF } \\
n=53 \\
(27.3 \%)\end{array}$ & $p$-value \\
\hline Age & $59.6(12.1)$ & $60.5(12.8)$ & $59.9(11.5)$ & $57.8(10.9)$ & $0.429^{a}$ \\
\hline \multicolumn{6}{|l|}{ Sex } \\
\hline Male & $140(72.2)$ & $62(61.4)$ & 35 (87.5) & $43(81.1)$ & $0.002^{b}$ \\
\hline Female & $54(27.8)$ & $39(38.6)$ & $5(12.5)$ & $10(18.9)$ & \\
\hline \multicolumn{6}{|l|}{ Ethnicity } \\
\hline Malay & $78(40.2)$ & 39 (38.6) & $17(42.5)$ & $22(41.5)$ & $0.902^{\mathrm{b}}$ \\
\hline Chinese & $43(22.2)$ & $25(24.8)$ & $7(17.5)$ & $11(20.8)$ & \\
\hline Indian & $70(36.1)$ & $35(34.7)$ & $15(37.5)$ & $20(37.7)$ & \\
\hline Others & $3(1.5)$ & $2(2.0)$ & $1(2.5)$ & $0(0.0)$ & \\
\hline LVEF (\%) & $51.0(40-65)$ & $64(56-67)$ & $45(43-47)$ & $35(31.5-37)$ & $<0.001^{\mathrm{C}}$ \\
\hline \multicolumn{6}{|c|}{ NYHA Functional Class } \\
\hline Class I & $153(78.9)$ & 80 (79.2) & $32(80.0)$ & $41(77.4)$ & $0.097^{b}$ \\
\hline Class II & 35 (18.0) & 20 (19.9) & $8(20.0)$ & $7(13.2)$ & \\
\hline Class III & $5(2.6)$ & $1(1.0)$ & $0(0.0)$ & $4(7.5)$ & \\
\hline Class IV & $1(0.5)$ & $0(0.0)$ & $0(0.0)$ & $1(1.9)$ & \\
\hline $\mathrm{HbA1c}(\%)$ & $8.2(6.9-9.5) 8$ & $8.2(7.1-9.6)$ & $8.5(6.8-9.8)$ & $7.8(6.7-9.1)$ & $0.348^{c}$ \\
\hline \multicolumn{6}{|l|}{ Glycemic Control } \\
\hline $\mathrm{HbA} 1 \mathrm{c} \leq 7$ & $50(25.8)$ & $22(21.8)$ & $10(25.0)$ & $18(34.0)$ & $0.258^{\mathrm{b}}$ \\
\hline $\mathrm{HbA} 1 \mathrm{c}>7$ & $144(74.2)$ & 79 (78.2) & 30 (75.0) & 35 (66.0) & \\
\hline
\end{tabular}

Values are expressed as number (\%) for categorical data; mean \pm standard deviation, or median (interquartile range) for continuous data; ${ }^{a}$ computed by One-Way ANOVA; ${ }^{b}$ computed by Pearson's Chi-square test; ${ }^{c}$ computed by Kruskal Wallis test; bolded font indicates statistical significance at $p<0.05$. HbA1C, glycated hemoglobin; HFmrEF, heart failure with mid-range ejection fraction; HFpEF, heart failure with preserved ejection fraction; HFrEF, heart failure with reduced ejection fraction; LVEF, left ventricular ejection fraction; SGLT-2, sodium glucose linked transporter-2; NYHA, New York Heart Association.

LVEF was not normally distributed with a median of $51 \%$ (interquartile range $40-65 \%$ ). In the study population, more than half of the patients had LVEF of $\geq 50 \%$. Results revealed that more than three-quarters of patients were placed in NYHA Class I, followed by Class II, III, and IV. In all groups, more than three-quarters of patients were placed in NYHA Class 1. In this study, only one patient, under the HFrEF group, was assigned with NYHA Class IV. Median HbA1c was $8.2 \%$ (interquartile range 6.9-9.5\%). Approximately three-quarters $(74.2 \%)$ of patients had poor glycemic control with $\mathrm{HbA1c}$ of $>7$, categorized based on the American Diabetes Association recommendations. Among the three patient groups, a higher proportion of HFrEF patients had good glycemic control and had the lowest median HbA1c. There were no significant differences in terms of NYHA functional class and glycemic control across the three patient groups.

\section{Antihyperglycemic Agents Prescribed in HF Patients With T2DM}

Overall, metformin (59.8\%) was the most commonly prescribed antihyperglycemic agent, followed by insulins (56.2\%), and sulphonylureas (45.4\%), as shown in Table 2. In HFpEF patients, metformin (65.3\%) was most commonly prescribed, followed by insulins (60.4\%). In HFmrEF patients, insulins $(62.5 \%)$ were most commonly prescribed, followed by metformin (57.5\%). The most commonly prescribed agent for patients with HFrEF was sulphonylurea (60.4\%). More than $60 \%$ of patients from HFpEF and HFmrEF categories were prescribed with insulins.

Sulphonylurea was the only antihyperglycemic agent class found to be significantly associated with LVEF subgroups $(p=0.033)$. From Table 2, the odds for sulphonylurea prescription among the HFrEF patients were 2.42 times higher compared to the HFpEF patients, with a 95\% confidence interval of [1.23-4.79]. The HFrEF patients were less than half as likely to being prescribed with insulins compared to the HFpEF patients, with a 95\% confidence interval of [0.26-0.99]. Otherwise, there were no differences in terms of prescribing pattern of other agents across the three patient groups.

\section{Biguanides}

Metformin was prescribed in 116 patients for glycemic control. As shown in Figure 2, the most frequently prescribed regimen was metformin 2,000 mg daily, in 63 patients. Sixty-one patients were prescribed with immediate-release metformin $1 \mathrm{~g}$ twice daily, while two were prescribed with extended-release (ER) metformin at a dose of $2,000 \mathrm{mg}$ once daily. Two patients on metformin $1,500 \mathrm{mg}$ daily were both prescribed with oncedaily extended-release tablets. Out of 47 patients receiving metformin $1,000 \mathrm{mg}$ daily, five received metformin ER $1 \mathrm{~g}$ once daily while the remaining was on immediate-release metformin $500 \mathrm{mg}$ twice daily. A total of four patients received metformin $500 \mathrm{mg}$ daily, with two patients receiving metformin $250 \mathrm{mg}$ twice daily, and another two receiving metformin ER $500 \mathrm{mg}$ daily.

\section{Sulphonylureas}

A total of 88 patients were prescribed with gliclazide. Thirtysix patients $(40.9 \%)$ from them were prescribed with gliclazide modified release (MR) formulation.

\section{Dipeptidyl Peptidase-4 Inhibitors}

More than $80 \%$ of patients on DPP-4 inhibitors were on vildagliptin, with 12 patients on vildagliptin $50 \mathrm{mg}$ od, and 30 patients on vildagliptin $50 \mathrm{mg}$ bd; whereas the remaining eight patients were prescribed with sitagliptin in different doses of 25, 50 , or $100 \mathrm{mg}$ once daily.

\section{Sodium Glucose Linked Transporter-2 Inhibitors}

Only four patients were prescribed with SGLT-2 inhibitors. The dosage regimens used in these patients were once-daily empagliflozin $25,12.5$, and $10 \mathrm{mg}$.

\section{Insulins}

As shown in Figure 3, the subcutaneous basal-bolus regimen was the most commonly prescribed. Sixty-one out of 66 patients on a basal-bolus regimen were prescribed with a combination of intermediate-acting NPH insulin and three separate injections of short-acting regular insulin at each meal. The remaining five 
TABLE 2 | Antihyperglycemic agents prescribed in HF patients with T2DM.

\begin{tabular}{|c|c|c|c|c|c|}
\hline \multirow{2}{*}{$\begin{array}{l}\text { Classes of } \\
\text { antihyperglycemic } \\
\text { agents }\end{array}$} & \multicolumn{4}{|c|}{ Number of patients (\%) } & \multirow[t]{2}{*}{$p$-value ${ }^{b}$} \\
\hline & Total $^{\mathbf{a}}$ & $\begin{array}{c}\text { HFpEF } \\
(n=101)\end{array}$ & $\begin{array}{l}\text { HFmrEF } \\
(n=40)\end{array}$ & $\begin{array}{l}\text { HFrEF } \\
(n=53)\end{array}$ & \\
\hline \multicolumn{6}{|l|}{ Biguanides } \\
\hline Yes & $116(59.8)$ & $66(65.3)$ & $23(57.5)$ & $27(50.9)$ & 0.211 \\
\hline OR (95\% Cl) & & Reference & $0.72(0.34-1.52)$ & 0.55 (0.28-1.08) & \\
\hline \multicolumn{6}{|l|}{ Sulphonylureas } \\
\hline Yes & $88(45.4)$ & $39(38.6)$ & $17(42.5)$ & $32(60.4)$ & 0.033 \\
\hline OR (95\% Cl) & & Reference & $1.18(0.56-2.47)$ & 2.42 (1.23-4.79) & \\
\hline \multicolumn{6}{|l|}{ DPP-4 Inhibitors } \\
\hline Yes & $50(25.8)$ & $30(29.7)$ & 9 (22.5) & $11(20.8)$ & 0.420 \\
\hline OR (95\% Cl) & & Reference & $0.69(0.29-1.62)$ & $0.62(0.28-1.37)$ & \\
\hline \multicolumn{6}{|l|}{ SGLT2 Inhibitors } \\
\hline Yes & $4(2.1)$ & $1(1.0)$ & $1(2.5)$ & $2(3.8)$ & 0.501 \\
\hline OR $(95 \% \mathrm{Cl})$ & & Reference & $2.56(0.16-42.01)$ & $0.27(0.35-44.28)$ & \\
\hline \multicolumn{6}{|c|}{$\alpha$-glucosidase Inhibitors } \\
\hline Yes & $1(0.5)$ & $0(0.0)$ & $0(0.0)$ & $1(1.9)$ & 0.263 \\
\hline OR (95\% Cl) & & UTC & UTC & UTC & \\
\hline \multicolumn{6}{|l|}{ Insulins } \\
\hline Yes & 109 (56.2) & $61(60.4)$ & $25(62.5)$ & $23(43.4)$ & 0.086 \\
\hline OR (95\% Cl) & & Reference & 1.09 (0.51-2.32) & $0.50(0.26-0.99)$ & \\
\hline
\end{tabular}

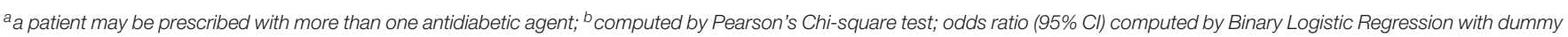

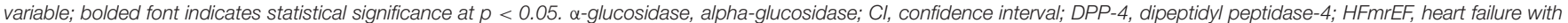

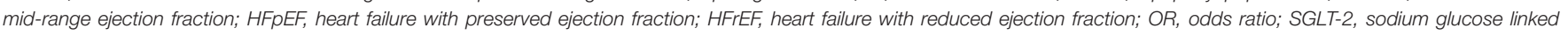
transporter-2, UTC, unable to compute.

\section{The Prescribing Pattern of Biguanides}

\section{Total Daily \\ Dosage of Metformin \\ Metformin 2000mg \\ Metformin 1500mg \\ Metformin 1000mg}

Metfromin 500mg

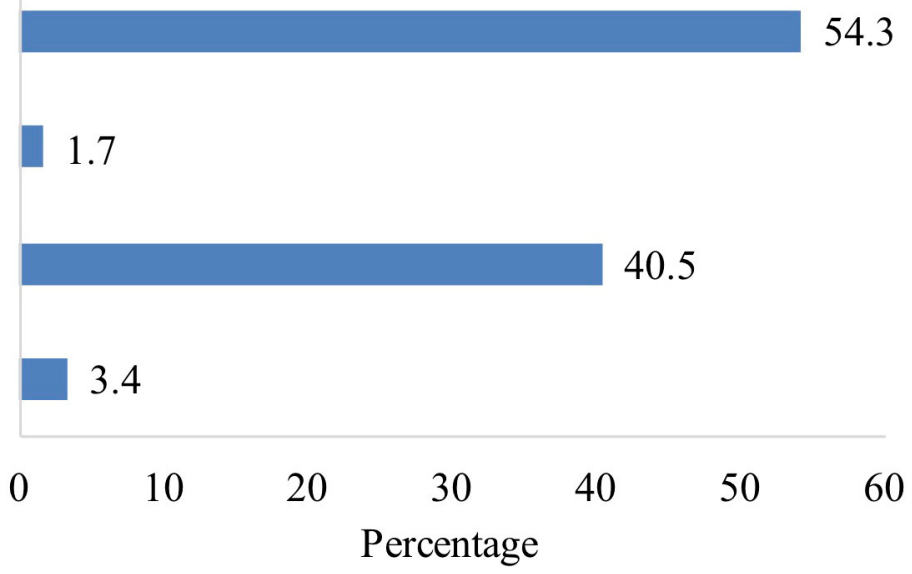

\section{3}

60

FIGURE 2 | Daily dosage regimens of metformin $(n=116)$.

patients were prescribed with long-acting basal insulin analogs. The second most frequently prescribed regimen was the twicedaily pre-mixed insulin. All of the 34 patients were prescribed with pre-mixed human insulin except for one who was given pre-mixed insulin analog. Of the seven patients receiving oncedaily insulin before bed, six received NPH insulin while one received insulin analog. Two other patients received multiple insulin injection regimens. Overall, more than $90 \%$ of patients 


\section{The Prescribing Pattern of Insulin Regimens and Therapies}

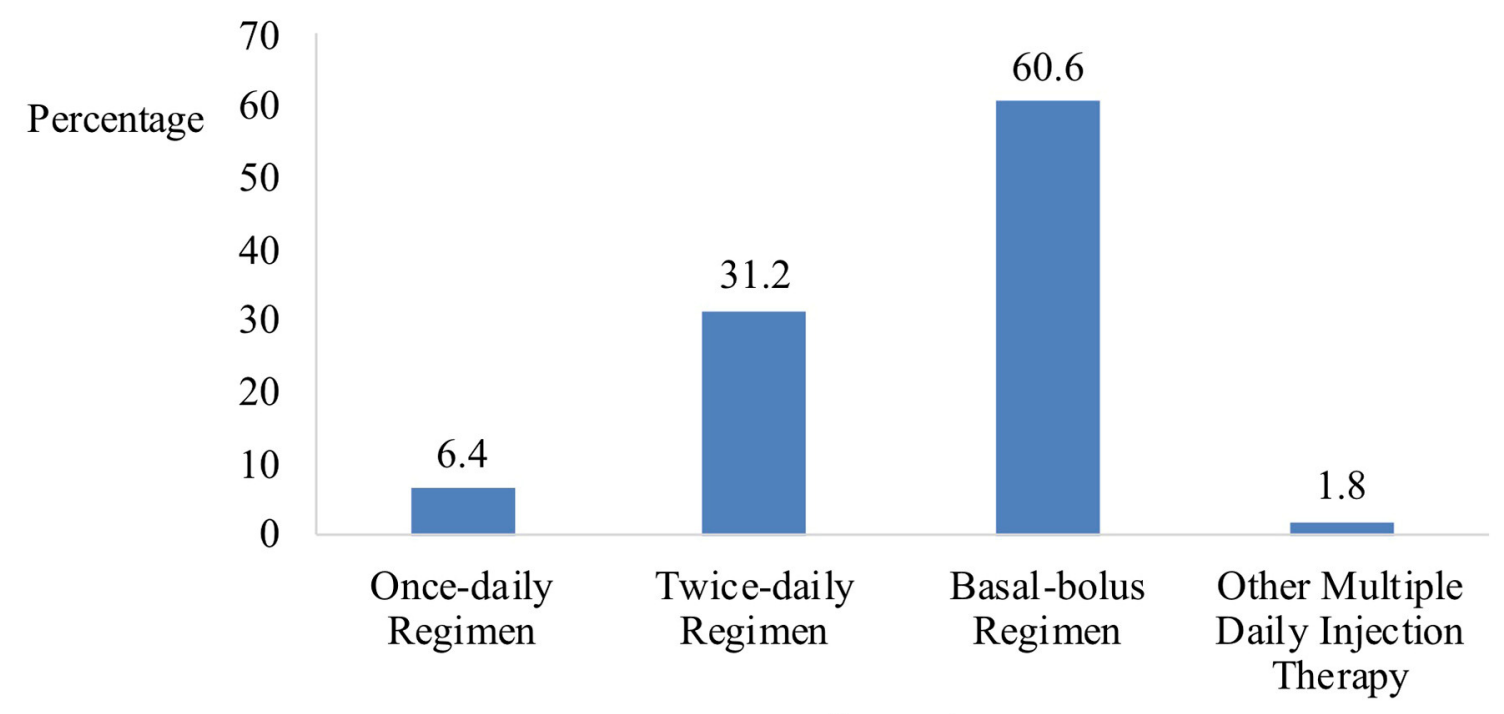

Insulin Regimens

FIGURE 3 | Daily Regimens of Insulins ( $n=109)$.

requiring insulin were prescribed with human insulins instead of insulin analogs.

\section{Associations of Patients Characteristics and Antihyperglycemic Agents With LVEF}

In Table 3, multiple Multinomial Logistic Regression models on the association between sulphonylurea prescribing trend and patients' characteristics with LVEF were displayed. In Model 1, being male was significantly associated to be in the HFmrEF and HFrEF subgroups with ORs of 4.40 (95\% CI 1.59-12.20) and 2.71 (95\% CI 1.22-6.00), respectively. In Model 2, unadjusted association between sulphonylurea and LVEF subgroups showed that those who were prescribed with sulphonylurea were 2.42 times more likely to be HFrEF patients. Both sulphonylurea prescription and male sex remained significantly associated with HFrEF subgroup even after adjusting for age and HbAlc in the subsequent models.

\section{DISCUSSION}

In our study cohort of patients with both HF and T2DM, metformin is the most prescribed antihyperglycemic agent. The prescribing trend of sulphonylureas was significantly associated with patients' LVEF status, where HFrEF patients were 2 times more likely to receive sulphonylurea compared to the HFpEF patients. The use of SGLT-2 inhibitors, the evidence-based medications associated with improved outcomes in HF with T2DM, was low at 2.1\%. There was no association found between glycemic control with patients' LVEF.
Metformin was the most utilized (59.8\%) antihyperglycemic agent as metformin was the recommended first-line medication in the current practice guidelines (15). Metformin was also the most commonly prescribed diabetes medication in the Asian Sudden Cardiac Death in Heart Failure (ASIAN-HF) registry, the Dapagliflozin And Prevention of Adverseoutcomes in Heart Failure (DAPA-HF) trial, and other studies across Asia, with prescription rates ranging from 50.8 to $56.8 \%(16-18)$. The prescription rates reported were similar to that in our study. In contrast, two individual Asian countries namely Japan and China reported low metformin use at 11.8 and $14.5 \%$, respectively, for reasons yet to be assessed (17). The European Society of Cardiology (ESC) Guidelines for heart failure reported prescribing of metformin to be safe for HF patients, taking into consideration the patient's age and degree of renal or hepatic dysfunction (1). Metformin is effective, safe, and associated with improved outcomes such as reduction in all-cause hospitalization and mortality in $\mathrm{HF}$ patients in the ASIAN-HF registry $(19,20)$. Therefore, in accordance with current guideline recommendations, metformin should remain as the first drug of choice unless contraindicated.

Sulphonylureas were the most prescribed antihyperglycemic class in $60.4 \%$ of patients with HFrEF in our study. The usage of sulphonylurea was frequent despite being associated with hypoglycemia, weight gain, and increased risk of worsening HF (1, 21). Across Asia, the use of sulphonylurea therapies among HFrEF patients ranged from 43.8 to $53 \%(17,18)$. The high prescription rate of sulphonylurea, despite lack of benefits on outcomes, might be due to other factors not examined in our study, such as the presence of renal impairment 
TABLE 3 | Patients characteristics and antihyperglycemic agents associated with LVEF.

\begin{tabular}{|c|c|c|c|}
\hline & \multicolumn{3}{|c|}{ Odds Ratio $(95 \% \mathrm{Cl})$} \\
\hline & HFpEF & HFmrEF & HFrEF \\
\hline \multicolumn{4}{|l|}{ Model 1} \\
\hline Male Sex & Reference & $4.40(1.59-12.20)$ & $2.71(1.22-6.00)$ \\
\hline \multicolumn{4}{|l|}{ Model 2} \\
\hline Sulphonylurea & Reference & $1.18(0.56-2.47)$ & $2.42(1.23-4.79)$ \\
\hline \multicolumn{4}{|l|}{ Model 3} \\
\hline Male Sex & Reference & 4.40 (1.58-12.25) & $2.51(1.11-5.66)$ \\
\hline Sulphonylurea & Reference & $1.04(0.48-2.23)$ & $2.22(1.11-4.45)$ \\
\hline Age & Reference & $0.99(0.96-1.03)$ & $0.98(0.95-1.01)$ \\
\hline \multicolumn{4}{|l|}{ Model 4} \\
\hline Male Sex & Reference & $4.78(1.69-13.50)$ & $2.35(1.03-5.39)$ \\
\hline Sulphonylurea & Reference & $1.07(0.49-2.30)$ & $2.17(1.08-4.36)$ \\
\hline Age & Reference & $0.99(0.97-1.03)$ & $0.98(0.95-1.01)$ \\
\hline $\mathrm{HbA1c}$ & Reference & $1.11(0.93-1.31)$ & $0.94(0.79-1.11)$ \\
\hline
\end{tabular}

or hepatic disease, where metformin use is contraindicated. Although the ADVANCE trial had reported no difference in $\mathrm{HF}$ hospitalization rate in patients randomized to nosulphonylurea group or gliclazide-combination group, other studies have found unfavorable risk profile of sulphonylurea when compared with metformin, with sulphonylurea being associated with higher risk of all-cause mortality and congestive HF $(22,23)$. Hence, a shift from sulphonylurea to metformin prescription should be considered over time. However, if adequate glycemic control cannot be achieved with metformin or other classes of antihyperglycemic agent with regards to contraindications, co-morbidities, or relative cost, sulphonylurea could be used with caution and close monitoring. In addition, patients receiving gliclazide should be given gliclazide modified release (MR) formulation as its once-daily dosing regimen helps to enhance patient's adherence to medication, thus resulting in better blood glucose control when compared to conventional gliclazide.

There was a strikingly under-usage of SGLT-2 inhibitors in our study cohort with a prescription rate of $2.1 \%$, in contrast to $26.5 \%$ in Taiwan as reported by Chang HY et al. (18). Although Chang HY et al. reported a higher prescription rate, SGLT2 inhibitor was only the fourth most commonly prescribed antihyperglycemic agent after metformin, Dpp-4 inhibitor, and sulphonylurea in their cohort of heart failure patients (18). Reason for the infrequent use of SGLT-2 inhibitors in our study cohort could be related to higher cost and limited drug formulary availability. The cost of SGLT-2 inhibitors had also severely limits its availability in other developing countries within Asia (20). The DAPA-HF trial, EMPA-REG OUTCOME trial (Empagliflozin Cardiovascular Outcome Event Trial in Type 2 Diabetes Mellitus Patients), and CANVAS (Canagliflozin Cardiovascular Assessment Study) confirmed that SGLT2- inhibitor treatment lowered rates of heart failure hospitalization (24-26). Because of the consistent cardiovascular benefits demonstrated among both the Asians and non-Asians, it is important to consider escalating the use of SGLT-2 inhibitors in HF patients with T2DM.

In this study, glycemic control was not found to be associated with LVEF. This stands in contrast to the results from a cohort study in a region of South China which observed a curved, U-shaped correlation between HbA1c levels with LV diastolic dysfunction $(2,27)$. Our study has shown that male was independently associated with lower LVEF. This sex-related differences was consistent with findings from the Registry to Improve the Use of Evidence-Based Heart Failure Therapies in the Outpatient Setting (IMPROVE HF) which revealed that females were significantly associated with improvement in LVEF when compared to males (28). The result from our study indicated that LVEF might be affected by other confounding factors not examined in our study such as BMI, smoking status, co-morbidities or heart failure medications and their doses.

\section{Study Strength and Limitation}

In this study, real-time data such as antihyperglycemic agents, glycemic control, patients' HF classification according to both LVEF and NYHA functional class were collected and analyzed, reflecting clinical practice in the actual situation. The exposure duration of patients to antihyperglycemic agents was welldefined, which was at least 3 months. Research site was selected based on patient population served, HF patient volume, and availability of expertise in echocardiography. There were several limitations in this study. Sampling of subjects from only one hospital implicates that the demonstrated characteristics of this study population might not accurately represent the entire Malaysian population. Also, due to the retrospective nature of the study design, there were incomplete information and missing data from some of the patients' medical records, such as the duration of HF and T2DM, as well as patients' renal and hepatic functions.

\section{CONCLUSION}

The prescribing patterns of antihyperglycemic agents varied amongst the three LVEF subgroups. Metformin was the most commonly prescribed antihyperglycemic agent. SGLT2 inhibitor was under-prescribed despite the Malaysian CPG recommendations and evidence that SGLT-2 inhibitor was related to improved outcomes in patients with established HF and DM. Our study also detected poorly-control diabetes presented in the vast majority of patients with heart failure. Understanding the current prescribing pattern of antihyperglycemic agents would help raise awareness of the importance of evidencebased treatment strategies in HF patients with T2DM to improve patients' outcomes. As both HF and DM are chronic disorders, HF patients with T2DM should be managed with close monitoring on safety and efficacy of antihyperglycemic therapies.

Data are scarce regarding the co-disease management strategies and clinical outcomes in HF patients with DM in Malaysia. A prospective follow-up study on LVEF changes and cardiovascular outcomes of hospitalization and mortality in this study population is ongoing to address these gaps. It is 
hope that the result from the follow-up study will provide further insight into the long-term cardioprotective effects of glucose-lowering medications, thereby enhancing diabetes and heart failure pharmacological management, and optimizing both quality and quantity of life in HF patients with T2DM.

\section{DATA AVAILABILITY STATEMENT}

The raw data supporting the conclusions of this article will be made available by the authors, without undue reservation.

\section{ETHICS STATEMENT}

The studies involving human participants were reviewed and approved by The Medical Research and Ethics Committee (MREC), Ministry of Health Malaysia. Written informed consent for participation was not required for this study in accordance with the national legislation and the institutional requirements.

\section{REFERENCES}

1. Ponikowski P, Voors AA, Anker SD, Bueno H, Cleland JG, Coats AJ, et al. 2016 ESC Guidelines for the diagnosis and treatment of acute and chronic heart failure: the task force for the diagnosis and treatment of acute and chronic heart failure of the European Society of Cardiology (ESC). Developed with the special contribution of the Heart Failure Association (HFA) of the ESC. Eur J Heart Fail. (2016) 18:891-975. doi: 10.1002/ejhf.592

2. Dunlay SM, Givertz MM, Aguilar D, Allen LA, Chan M, Desai AS, et al. Type 2 diabetes mellitus and heart failure: a scientific statement from the american heart association and the heart failure society of America: this statement does not represent an update of the 2017 ACC/AHA/HFSA heart failure guideline update. Circulation. (2019) 140:e294-324. doi: 10.1161/CIR.0000000000000691

3. Malaysia MoH. Clinical Practice Guidelines on Management of Heart Failure. Kuala Lumpur: National Heart Association of Malaysia (2019).

4. Health IfP. National Health and Morbidity Survey 2011 (NHMS 2011). Kuala Lumpur: Malaysia Ministry of Health (2011).

5. Malaysia IfPH. National Health \& Morbidity Survey (NHMS 2015). Vol. II: Non-Communicable Diseases, Risk Factors \& Other Health Problems. Institute for Public Health Malaysia Kuala Lumpur (2015).

6. Gustafsson I, Brendorp B, Seibæk M, Burchardt H, Hildebrandt P, Køber L, et al. Influence of diabetes and diabetes-gender interaction on the risk of death in patients hospitalized with congestive heart failure. J Am Coll Cardiol. (2004) 43:771-7. doi: 10.1016/j.jacc.2003.11.024

7. Kannel WB, McGee DL. Diabetes and cardiovascular disease: the Framingham study. JAMA. (1979) 241:2035-8. doi: 10.1001/jama.241.19.2035

8. Greenberg BH, Abraham WT, Albert NM, Chiswell K, Clare R, Stough WG, et al. Influence of diabetes on characteristics and outcomes in patients hospitalized with heart failure: a report from the organized program to initiate lifesaving treatment in hospitalized patients with heart failure (OPTIMIZEHF). Am Heart J. (2007) 154:277.e1-8. doi: 10.1016/j.ahj.2007.08.002

9. Sarma S, Mentz RJ, Kwasny MJ, Fought AJ, Huffman M, Subacius H, et al. Association between diabetes mellitus and post-discharge outcomes in patients hospitalized with heart failure: findings from the EVEREST trial. Eur J Heart Fail. (2013) 15:194-202. doi: 10.1093/eurjhf/hfs153

10. Scirica BM, Braunwald E, Raz I, Cavender MA, Morrow DA, Jarolim $\mathrm{P}$, et al. Heart failure, saxagliptin, and diabetes mellitus: observations from the SAVOR-TIMI 53 randomized trial. Circulation. (2014) 130:157988. doi: 10.1161/CIRCULATIONAHA.114.010389

11. Kramer DG, Trikalinos TA, Kent DM, Antonopoulos GV, Konstam MA, Udelson JE. Quantitative evaluation of drug or device effects on ventricular remodeling as predictors of therapeutic effects on mortality in patients with

\section{AUTHOR CONTRIBUTIONS}

SL, HZ, and KC conceived the presented idea. SL developed the methodology and performed the computations. SL and KP collected the data. HZ and KC vetted the study design, data and statistics generated, references, and verified the analytical methods. ST aided in interpreting the results and worked on the manuscript. All authors discussed the results and contributed to the final manuscript.

\section{ACKNOWLEDGMENTS}

The authors would like to thank the Endocrine team, physicians, pharmacists, administrative staffs, and our colleagues in the HTAR Medical Department for their guidance, assistance, and technical support throughout the study. We would also like to thank the Director General of Health Malaysia for his permission to publish this article. heart failure and reduced ejection fraction: a meta-analytic approach. J Am Coll Cardiol. (2010) 56:392-406. doi: 10.1016/j.jacc.2010.05.011

12. Lincoff AM, Wolski K, Nicholls SJ, Nissen SE. Pioglitazone and risk of cardiovascular events in patients with type 2 diabetes mellitus: a meta-analysis of randomized trials. JAMA. (2007) 298:1180-8. doi: 10.1001/jama.298.10.1180

13. Sinha B, Ghosal S. Meta-analyses of the effects of DPP-4 inhibitors, SGLT2 inhibitors and GLP1 receptor analogues on cardiovascular death, myocardial infarction, stroke and hospitalization for heart failure. Diabetes Res Clin Pract. (2019) 150:8-16. doi: 10.1016/j.diabres.2019.02.014

14. Dean A, Sullivan K, Soe M. OpenEpi: Open Source Epidemiologic Statistics for Public Health, Version 3.01. (2014). Available online at: http://www.openepi. com/Menu/OE_Menu.htm (accessed Jul 1, 2019).

15. MalaysiaMoH. Clinical Practice Guidelines on Management of Type 2 Diabetes Mellitus. 5th ed. Putrajaya: Ministry of Health (2015).

16. McMurray JJ, DeMets DL, Inzucchi SE, Køber L, Kosiborod MN, Langkilde $\mathrm{AM}$, et al. The dapagliflozin and prevention of adverse-outcomes in heart failure (DAPA-HF) trial: baseline characteristics. Eur J Heart Fail. (2019) 21:1402-11. doi: 10.1002/ejhf.1548

17. Chia YM, Teng T, Tay WT, Anand I, MacDonald MR, Yap J, et al. Prescription patterns of anti-diabetic medications and clinical outcomes in Asian patients with heart failure and diabetes mellitus. Eur J Heart Fail. (2019) 21:6858. doi: 10.1002/ejhf.1467

18. Chang HY, Su YW, Feng AN, Fong MC, Huang KC, Chong E, et al. Prescription patterns of diabetes medications influencing clinical outcomes of heart failure patients with reduced ejection fraction. ESC Heart Fail. (2020) 7:604-15. doi: 10.1002/ehf2.12617

19. Eurich DT, Weir DL, Majumdar SR, Tsuyuki RT, Johnson JA, Tjosvold $\mathrm{L}$, et al. Comparative safety and effectiveness of metformin in patients with diabetes mellitus and heart failure: systematic review of observational studies involving 34000 patients. Circ Heart Fail. (2013) 6:395-402. doi: 10.1161/CIRCHEARTFAILURE.112.000162

20. Chia YMF, Teng THK, Chandramouli C, Yap J, MacDonald M, Lam CS. Clinical correlates and pharmacological management of Asian patients with concomitant diabetes mellitus and heart failure. Heart Fail Rev. (2018) 23:4618. doi: 10.1007/s10741-018-9672-5

21. Singh JS, Fathi A, Vickneson K, Mordi I, Mohan M, Houston JG, et al. Research into the effect Of SGLT2 inhibition on left ventricular remodelling in patients with heart failure and diabetes mellitus (REFORM) trial rationale and design. Cardiovasc Diabetol. (2016) 15:97. doi: 10.1186/s12933-016-0419-0

22. Tzoulaki I, Molokhia M, Curcin V, Little MP, Millett CJ, Ng A, et al. Risk of cardiovascular disease and all cause mortality among patients with type 2 diabetes prescribed oral antidiabetes drugs: retrospective 
cohort study using UK general practice research database. BMJ. (2009) 339:b4731. doi: 10.1136/bmj.b4731

23. ADVANCE Collaborative Group. Intensive blood glucose control and vascular outcomes in patients with type 2 diabetes. $N$ Engl J Med. (2008) 358:2560-72. doi: 10.1056/NEJMoa0802987

24. McMurray JJV, Solomon SD, Docherty KF, Jhund PS. The Dapagliflozin and prevention of adverse outcomes in heart failure trial (DAPA-HF) in context. Eur Heart J. (2020). doi: 10.1093/eurheartj/ehz916. [Epub ahead of print].

25. Fitchett D, Inzucchi SE, Cannon CP, McGuire DK, Scirica $\mathrm{BM}$, Johansen $\mathrm{OE}$, et al. Empagliflozin reduced mortality and hospitalization for heart failure across the spectrum of cardiovascular risk in the EMPA-REG OUTCOME trial. Circulation. (2019) 139:1384-95. doi: 10.1161/CIRCULATIONAHA.118.037778

26. Rådholm K, Figtree G, Perkovic V, Solomon SD, Mahaffey KW, de Zeeuw D, et al. Canagliflozin and heart failure in type 2 diabetes mellitus: results from the CANVAS Program. Circulation. (2018) 138:45868. doi: 10.1161/CIRCULATIONAHA.118.034222

27. Zuo X, Liu X, Chen R, Ou H, Lai J, Zhang Y, et al. An in-depth analysis of glycosylated haemoglobin level, body mass index and left ventricular diastolic dysfunction in patients with type 2 diabetes. BMC Endocr Disord. (2019) 19:88. doi: 10.1186/s12902-019-0419-7

28. Wilcox JE, Fonarow GC, Yancy CW, Albert NM, Curtis AB, Heywood JT, et al. Factors associated with improvement in ejection fraction in clinical practice among patients with heart failure: findings from IMPROVE HF. Am Heart J. (2012) 163:49-56.e2. doi: 10.1016/j.ahj.2011. 10.001

Conflict of Interest: The authors declare that the research was conducted in the absence of any commercial or financial relationships that could be construed as a potential conflict of interest.

Copyright $\odot 2020$ Lin, Phang, Toh, Chee and Zaman Huri. This is an open-access article distributed under the terms of the Creative Commons Attribution License (CC $B Y)$. The use, distribution or reproduction in other forums is permitted, provided the original author(s) and the copyright owner(s) are credited and that the original publication in this journal is cited, in accordance with accepted academic practice. No use, distribution or reproduction is permitted which does not comply with these terms. 